\title{
Free-breathing non-contrast MRA with efficiency-adaptive self navigation
}

\author{
Yibin Xie ${ }^{1,2^{*}}$, Zhaoyang Fan ${ }^{1}$, Rola Saouaf ${ }^{1}$, Yutaka Natsuaki ${ }^{3}$, Gerhard Laub³ ${ }^{3}$ Debiao Li,2 \\ From 16th Annual SCMR Scientific Sessions \\ San Francisco, CA, USA. 31 January - 3 February 2013
}

\section{Background}

Non-contrast MRA (NC-MRA) based on bSSFP and slab-selective inversion has become an attractive alternative for imaging renal arteries without the usage of potentially nephrotoxic contrast agent. It typically requires navigator gating or abdominal bellow triggering to alleviate breathing motion artifacts. However, navigator gating significantly complicates and lengthens exams due to its setup, adjusting, and scout scans. It also causes signal loss in renal arteries due to cross-pair saturation bands. Abdominal bellow triggering increases

patient preparation time and disables the usage of ECG triggering leading to suboptimal inflow effect. In this work, a novel self navigation (SN) technique is developed in an attempt to overcome the limitations of the aforementioned free-breathing methods while maintaining scanning efficiency.

\section{Methods}

An SN readout line modified to superior-inferior (SI) direction without partition or phase encoding is inserted at the end of each 3D bSSFP readout block (Fig. 1A).

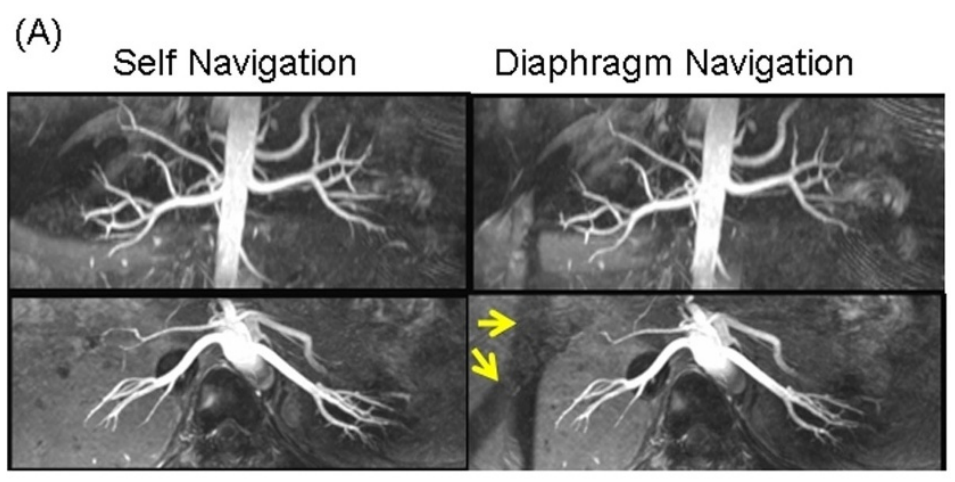

(B)

\begin{tabular}{|c|c|c|c|}
\hline & SNR & CNR & Score (5) \\
\hline Self Nav. & $44.7 \pm 7.3$ & $31.2 \pm 5.8$ & $4.67 \pm 0.71$ \\
\hline Diaphragm Nav. & $41.3 \pm 10.7$ & $28.2 \pm 8.7$ & $4.56 \pm 0.73$ \\
\hline T-test & 0.08 & 0.13 & 0.35 \\
\hline
\end{tabular}

Figure 1 (A) MIP images of self-navigated (left column) and conventional (right column) renal NC-MRA showing similar image quality. Yellow arrows point to saturation bands caused by conventional navigators. (B) Image quality comparison statistics.

${ }^{1}$ Cedars Sinai Medical Center, Los Angeles, CA, USA

Full list of author information is available at the end of the article 


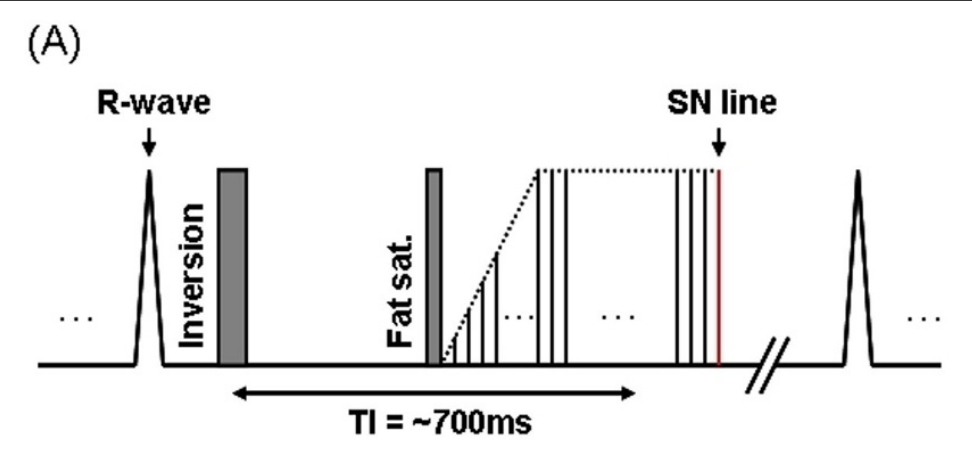

(B)

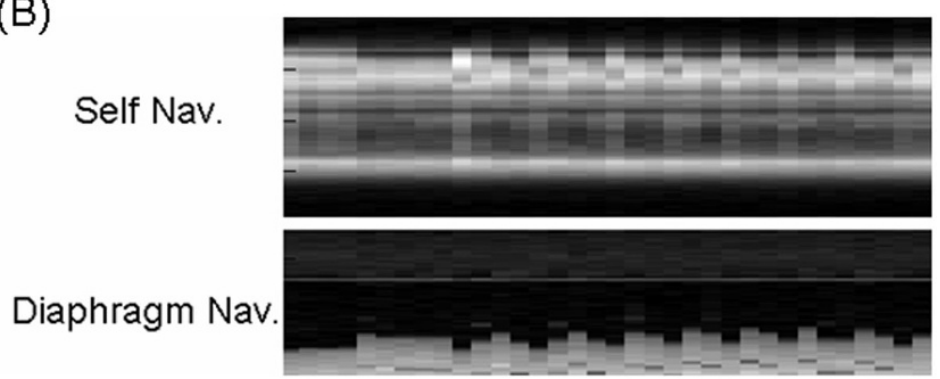

Figure 2 (A) Pulse diagram of SN NC-MRA. (B) Representative SN profiles (top) and diaphragm Navigator signal (bottom) showing high agreement.

Fourier transform of the $\mathrm{SN}$ readout line is the 1D projection of the entire imaging slab in the SI direction, which serves as the 'fingerprint' of the current respiratory phase. The reference projection profile is defined in the first two repetitions in the scan with breath-hold. In each subsequent repetition, the correlation coefficient (CC) is calculated between current projection profile and the reference profile. Respiratory motion is detected if the $\mathrm{CC}$ value drops below the threshold and current image lines will be rejected and reacquired in the next repetition. The threshold is dynamically adapted to maintain scanning efficiency.

Nine healthy volunteers with IRB approval were scanned on a 3T clinical scanner (MAGNETOM Verio, Siemens) with the following scan parameters: repetition $=700-900 \mathrm{~ms}$; TI $=550-750 \mathrm{~ms}$; acquisition time $=4-6$ min depending on subject heart rate; TE/TR $=1.9 / 3.8$ ms; 3D transverse slab with left-right readout; FOV = $400 \times 250 \mathrm{~mm} 2$, matrix $=304 \times 192$, slice thickness $=2.2$ (1.1 interpolated) $\mathrm{mm}$, yielding isotropic resolution = $1.1 \mathrm{~mm} 3$; $\mathrm{iPAT}=2$; bandwidth $=780 \mathrm{~Hz} /$ pixel; FA $=$ 90. For comparison, conventional navigator gated bSSFP MRA images were acquired immediately afterwards using same parameters.

\section{Results}

SN projections clearly show the underlining respiratory motion and are highly matched with diaphragm navigator (Fig. 1B). Excellent depiction of the intra- and extrarenal arteries are achieved using SN with no navigator saturation bands (Fig. 2A). No statistically significant difference was found between the two gating methods regarding SNR and CNR, as well as qualitative reviewer scores (Figure. 2B).

\section{Conclusions}

Preliminary results of SN bSSFP NC-MRA have demonstrated comparable image quality to conventional navigator gated acquisition but much simplified imaging planning and absence of saturation bands. Its performance in patients is currently under investigation.

\section{Funding}

This work is funded by grant NIH/NHLBI R01HL096119.

\section{Author details}

${ }^{1}$ Cedars Sinai Medical Center, Los Angeles, CA, USA. 'University of California, Los Angeles, Los Angeles, CA, USA. ${ }^{3}$ Siemens Healthcare, Los Angeles, CA, USA.

Published: 30 January 2013

doi:10.1186/1532-429X-15-S1-P233

Cite this article as: Xie et al:: Free-breathing non-contrast MRA with efficiency-adaptive self navigation. Journal of Cardiovascular Magnetic Resonance 2013 15(Suppl 1):P233. 